\title{
Les dispositifs juridiques de prévention des risques majeurs naturels en France
}

Pierre Tifine

\section{(2) OpenEdition}

\section{Journals}

Édition électronique

URL : http://journals.openedition.org/rge/4567

DOI : $10.4000 /$ rge.4567

ISSN : 2108-6478

Éditeur

Association des géographes de l'Est

Édition imprimée

Date de publication : 31 août 2013

ISSN : 0035-3213

Référence électronique

Pierre Tifine, "Les dispositifs juridiques de prévention des risques majeurs naturels en France », Revue Géographique de l'Est [En ligne], vol. 53 / 1-2 | 2013, mis en ligne le 20 septembre 2013, consulté le 10 décembre 2020. URL : http://journals.openedition.org/rge/4567 ; DOI : https://doi.org/10.4000/rge. 4567

Ce document a été généré automatiquement le 10 décembre 2020.

Tous droits réservés 


\title{
Les dispositifs juridiques de prévention des risques majeurs naturels en France
}

\author{
Pierre Tifine
}

Le rapport Détraigne publié en 2003 dans le cadre de l'élaboration du projet de loi relatif à la prévention des risques technologiques et naturels et à la réparation des dommages a procédé à une recension à peu près complète des risques naturels auxquels sont exposées les collectivités territoriales françaises ${ }^{1}$. Aujourd'hui, avec des gravités très variables, 23500 communes sur 36500 , sont exposées à un ou plusieurs risques naturels qu'il s'agisse de cyclones et de tempêtes, d'inondations, d'avalanches, de mouvements de terrain, de tremblements de terre, d'éruptions volcaniques, ou de feux de forêt. D'un point de vue statistique ces risques sont davantage présents dans les collectivités d'Outre-mer qu'en métropole. Le risque le plus fréquent est le risque d'inondation avec 15700 communes exposées, le risque de glissements de terrains (5 932 communes), de tremblements de terre (5 100 communes), le risque sismique (1 400 communes) et le risque d'avalanches ( 400 communes).

L'appréhension par le droit de ces risques naturels est ancienne. Déjà, l'article $5 \mathrm{du}$ Titre XI de la loi des 16 et 24 août 1790 donnait compétence aux autorités municipales pour « prévenir et faire cesser les accidents et les fléaux calamiteux ». A partir de la fin $\mathrm{du} \mathrm{XVIII}^{\circ}$ siècle et surtout au $\mathrm{XIX}^{\circ}$ l'industrialisation et un certain nombre de catastrophes qui se sont produites à l'époque on d'abord conduit le législateur à s'intéresser aux risques d'origine anthropiques's. La question des risques naturels ne se posera dans un premier temps que sur des questions ponctuelles. En particulier, les lois du 28 août 1860 sur le reboisement en montagne et du 4 avril 1882 sur la restauration et la conservation des terrains en montagne contiennent des dispositions relatives à la lutte contre l'érosion et à la prévention des risques de glissement de terrain, de crue torrentielle et d'avalanches. Par la suite un décret-loi du 30 octobre 1935 a créé les plans de surface submersible en vue d'assurer la gestion des risques d'inondation. Ces plans sont à l'origine directe des actuels plans de prévention des risques naturels prévisibles. 
De nos jours, les risques naturels - comme les risques technologiques - doivent être pris en compte par les documents d'urbanisme, c'est-à-dire par les documents qui ont vocation à préciser zone par zone les grandes options d'aménagement définies par les autorités compétentes. Sont concernés les schémas de cohérence territoriale ${ }^{3}$, qui s'appliquent à l'échelle de plusieurs communes ou groupements de communes, et surtout les plans locaux d'urbanisme qui vont déterminer, à l'échelon communal, la constructibilité des différentes zones qu'ils définissent ${ }^{4}$. Ces documents sont réalisés par les établissements publics de coopération territoriale, pour ce qui concerne les schémas de cohérence territoriale, et par les communes pour ce qui concerne les plans locaux d'urbanisme. Dans les deux cas il s'agit de personnes publiques locales distinctes de l'Etat. Le pouvoir central n'est toutefois pas démuni face à des collectivités qui par négligence ou par esprit mercantile négligeraient de prendre en compte les risques naturels et technologiques. En effet, conformément aux dispositions de l'article L. 123-14 du Code de l'urbanisme, le préfet peut imposer aux communes de modifier leur plan local d'urbanisme, au regard de l'existence de risques naturels ou technologiques en imposant le respect du projet d'intérêt général qui est un document élaboré par l'Etat dont l'un des objectifs peut justement consister en la prévention des risques.

Tout les outils que l'on vient de mentionner peuvent être utilisés par les autorités locales et nationales pour la prévention des risques notamment naturels, mais il s'agit avant tout d'outils de planification de l'aménagement pour la mise en œuvre desquels la prévention des risques ne constitue que l'un des paramètres à prendre en compte. Ce qui nous intéressera ici, plus spécifiquement, ce sont les instruments exclusivement dédiés à la prévention des risques naturels, lesquels peuvent être deux ordres : certains concernent la prévention des risques naturels en général (I) alors que d'autres régissent la prévention de certaines catégories de risques naturels envisagés isolément (II).

\section{Le cadre général de la prévention des risques naturels}

Le cadre général de la prévention des risques naturels est constitué par des plans (A) et par la possibilité qui est reconnue aux autorités publiques d'utiliser un certain nombre de pouvoirs qui leur sont reconnus (B).

\section{A. Les outils de planification}

La loi Barnier du 2 février 19955, actuellement codifiée dans le Code de l'environnement ${ }^{6}$, a remplacé les anciens plans d'exposition aux risques ${ }^{7}$, les plans de surfaces submersibles, les plans de zones sensibles aux incendies de forêt et les périmètres de risque par un outil unique : les plans de prévention des risques naturels prévisibles $\left(1^{\circ}\right)$. Par la suite, la loi du 30 juillet 2003 relative à la prévention des risques technologiques et naturels et à la réparation des dommages ${ }^{8}$ a créé les schémas de prévention des risques naturels $\left(2^{\circ}\right)$. 


\section{$1^{\circ}$. Les plans de prévention des risques naturels prévisibles}

Ces plans constituent les principaux outils juridiques de prévention desrisques naturels. Dans son rapport de 2010 le Conseil d'Etat a ainsi relevé que sur les 17064 communes, couvrant $46 \%$ du territoire, qui sont exposées à des risques naturels, 7747 sont dotées d'un PPRNP approuvé9.

La procédure d'élaboration du plan devra associer les communes et les établissements publics de coopération communale. Plus précisément, une enquête publique, au cours de laquelle sont entendus, après avis de leur conseil municipal, les maires des communes sur le territoire desquelles le plan doit s'appliquer doit être diligentée ${ }^{10}$. Cette enquête permet également à toute personne intéressée de consigner ses observations sur un registre spécialement tenu à cet effet.

C'est le préfet qui est compétent pour arrêter ce plan, voire plusieurs préfets qui devront prendre un arrêté conjoint lorsque le plan s'applique sur le territoire de plusieurs départements ${ }^{11}$. Le plan approuvé vaut servitude d'utilité publique et il est annexé au plan local d'urbanisme ${ }^{12}$.

Cette procédure est relativement longue et c'est pour cela que l'article L. 562-2 du Code de l'environnement prévoit que si l'urgence le justifie, le préfet peut, après consultation des maires concernés, décider de rendre certaines dispositions du plan immédiatement opposables. Ceci permet, par exemple, de faire immédiatement obstacle au développement de l'urbanisation dans les zones à risques ${ }^{13}$.

Si cela s'avère nécessaire, un même plan peut concerner plusieurs risques. On peut ici mentionner une circulaire interministérielle du 20 juillet 2006 qui recommande l'élaboration de ce type de plans en zones littorales en vue de traiter en même temps des risques d'inondation et de mouvements de terrain ${ }^{14}$.

Sur la façon de délimiter les zones exposées aux risques, l'article L. 562-1 du Code de l'environnement se borne à indiquer que l'autorité préfectorale doit tenir compte de l'intensité du risque encouru. Il en résulte une grande liberté dans les modalités de détermination des zones à risque. Ainsi, il a été jugé qu'aucun texte n'imposait de recourir à une méthode particulière pour définir le zonage ${ }^{15}$. Par exemple, le Conseil d'Etat a pu considérer qu'il était possible, en vue de déterminer l'intensité de l'aléa, de faire reconstituer la ligne de crue selon une méthode hydro-géomorphologique ${ }^{16}$.

Le plan lui-même va distinguer deux grands types de zones: les zones exposées aux risques, appelées "zones de danger ", et les "zones de précaution ", qui ne sont pas directement exposées aux risques mais dans lesquelles des constructions, des ouvrages, des aménagements ou des exploitations pourraient aggraver des risques ou en provoquer de nouveaux ${ }^{17}$. Le fait de construire ou d'aménager un terrain dans une zone interdite par le plan ou de ne pas respecter les conditions de réalisation, d'utilisation ou d'exploitation prescrites par ce plan est puni des peines qui sont prévues aux articles L. 480-4 du Code de l'urbanisme auquel renvoie l'article L. 562-5 du Code de l'environnement.

En dehors du zonage le plan définit les mesures de prévention, de protection et de sauvegarde qui doivent être prises, dans les zones de danger et de précaution, par les collectivités publiques et/ou par les particuliers ${ }^{18}$. A titre d'exemple, le plan peut imposer aux particuliers la réalisation de travaux de prévention des risques ainsi que la gestion de dispositifs de prévention ou d'intervention en cas de réalisation du risque. Le préfet dispose de pouvoirs de contrainte lui permettant d'assurer le respect de ces 
prescriptions. Plus précisément, la réalisation des mesures prévues par le plan peut être rendue obligatoire en fonction de la nature et de l'intensité du risque dans un délai de cinq ans qui peut être réduit en cas d'urgence. Après une mise en demeure infructueuse, le préfet peut ordonner la réalisation de ces mesures aux frais du propriétaire, de l'exploitant ou de l'utilisateur ${ }^{19}$.

Lorsque le plan impose des obligations aux propriétaires, exploitants ou utilisateurs de biens existants, le coût des mesures exigées ne peut excéder $10 \%$ de la valeur vénale ou estimée du bien à la date d'approbation du plan ${ }^{20}$. Par ailleurs, lorsqu'elles sont obligatoires, ces mesures peuvent bénéficier d'un financement du Fonds de prévention des risques majeurs ${ }^{21}$.

Enfin, il faut relever que les servitudes créées par le plan ne donnent pas lieu à indemnisation, même lorsqu'elles portent atteinte à des droits acquis ${ }^{22}$. Toutefois, par extension de la jurisprudence Bitouzet ${ }^{23}$, il faut considérer que le propriétaire doit être indemnisé s'il supporte « une charge spéciale et exorbitante hors de proportion avec l'objectif d'intérêt général poursuivi " ${ }^{24}$. Mais il faut ici relever que cette possibilité n'a pas encore fait l'objet d'application concrète par le juge et elle avoir vocation à être d'application exceptionnelle.

\section{$2^{\circ}$. Les schémas de prévention des risques naturels}

Les schémas de prévention des risques naturels ont été créés par la loi $n^{\circ}$ 2003-699 $d u$ 30 juillet $2003^{25}$, relative à la prévention des risques technologiques et naturels et à la réparation des dommages. Selon l'article R. 561-5 du Code de l'environnement il s'agit de "documents d'orientation quinquennaux fixant des objectifs généraux à partir d'un bilan et définissant un programme d'actions ». Ce programme d'actions concerne selon l'article L. 565-2 du même code: la connaissance du risque ; la surveillance et prévision des phénomènes; l'information et l'éducation sur les risques; la prise en compte des risques dans l'aménagement du territoire ; la mise en ceuvre de travaux permettant de réduire le risque; des retours d'expériences.

Comme le plan de prévention des risques naturels prévisibles, le projet de schéma est établi par le préfet et soumis à l'avis de la commission départementale des risques naturels majeurs ${ }^{26}$. Il est ensuite approuvé par arrêté préfectoral. Le schéma peut être modifié selon une procédure identique ${ }^{27}$.

Par souci de transparence, le public a la possibilité de consulter le schéma approuvé en préfecture et dans les sous-préfectures ${ }^{28}$. Enfin, un rapport sur l'exécution du schéma est présenté chaque année à la commission départementale des risques naturels majeurs ${ }^{29}$.

\section{B. Les pouvoirs reconnus aux autorités publiques}

Confrontées à une situation de risques naturels, les autorités publiques peuvent utiliser différents instruments juridiques leur permettant d'agir avec une certaine rapidité : le maire peut prendre, dans le cadre de ses pouvoirs de police administrative générale, des mesures de sûreté en cas de péril, alors que l'administration peut s'approprier des immeubles exposés à des risques naturels majeurs. 


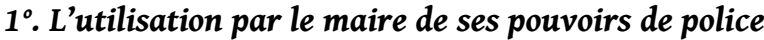

L'article L. 2212-5 $5^{\circ}$ du Code général des collectivités territoriales prévoit que la police municipale «comprend notamment le soin de prévenir, par des précautions convenables, et de faire cesser ... les incendies, les inondations, les ruptures de digues, les éboulements de terre ou de rochers, les avalanches ou autres accidents naturels ... de pourvoir d'urgence à toutes les mesures d'assistance et de secours et, s'il y a lieu, de provoquer l'intervention de l'administration supérieure». C'est donc un champ d'intervention très large qui est ouvert au maire qui peut également, selon l'article $\mathrm{L}$. 2212-4 du même code, en cas de danger grave ou imminent " prescrire l'exécution des mesures de sûreté exigées par les circonstances ». Ces mesures peuvent également être prises par le préfet, lorsque le site menacé s'étend sur plusieurs communes ou en cas de carence du maire.

En application de ces dispositions, l'autorité compétente peut ordonner l'évacuation d'un site lorsque les circonstances le justifient. Elle peut également ordonner la fermeture d'un établissement. Par exemple, il a été jugé qu'un préfet pouvait légalement, dans le cadre de ses pouvoirs de police administrative générale, décider de la fermeture d'un camping soumis «à raison de sa situation géographique à un risque élevé d'inondation par débordement torrentiel $\aleph^{30}$. Le maire, ou le préfet, peut également prescrire les travaux de nature à parer la survenance d'un risque naturel. Un maire peut ainsi légalement prendre un arrêté ordonnant au propriétaire du terrain de faire procéder d'urgence "aux travaux nécessaires afin de stopper le glissement en cours $~^{31}$. Cependant, dans la mesure où ces travaux présentent un intérêt collectif, ils doivent être exécutés par les soins de la commune et à ses frais.

De leur côté, les propriétaires ont également l'obligation de prendre toutes les mesures nécessaires de nature à éviter que leur fonds ne crée des risques sur les propriétés voisines. Ainsi, si la charge financière des travaux est supportée par la commune en cas de danger grave et imminent, celle-ci dispose d'un recours contre le propriétaire lorsque la situation lui est en partie imputable. Tel est le cas, par exemple, s'il apparaît que le propriétaire a reconnu ne pas avoir fait réaliser les travaux nécessaires à la stabilisation d'un terrain pour des raisons financières ${ }^{32}$.

Toutes les hypothèses que l'on vient de mentionner concernent l'utilisation par le maire ou par le préfet de pouvoirs de police administrative générale, lesquels se caractérisent par l'édiction de mesures préventives propre à faire cesser un trouble à l'ordre public, et plus précisément à la sécurité publique. Mais les pouvoirs de police ne sont pas cantonnés à cette seule hypothèse, puisque le maire dispose également d'un pouvoir de police administrative spéciale des édifices menaçant ruine qui trouve sa source non pas dans le Code général des collectivités territoriales, mais dans les articles L. 511-1 à L. 511-4 du Code de la construction et de l'habitation. Ce sont ces pouvoirs qui doivent être utilisés, conformément à la jurisprudence du Conseil d'Etat, lorsque le danger provoqué par un immeuble "provient à titre prépondérant de causes qui lui sont propres $»^{33}$. En revanche, c'est la police générale qui s'applique lorsque le danger menaçant l'immeuble a une cause extérieure à celui-ci, par exemple un effondrement du sous-sol à la suite d'une décompression du terrain ${ }^{34}$. Par ailleurs, la frontière entre police administratif générale et police spéciale des édifices menaçant ruine n'est pas toujours intangible. Le Conseil d'Etat a ainsi pu juger qu'en "présence d'une situation d'extrême urgence créant un péril particulièrement grave et imminent, le maire peut, quelle que soit la cause du danger, faire légalement usage de ses pouvoirs de police 
générale, et notamment prescrire l'exécution des mesures de sécurité qui sont nécessaires et appropriées $»^{35}$.

\section{$2^{\circ}$. L'appropriation publique d'immeubles exposés aux risques naturels}

L'appropriation publique d'immeubles exposés à des risques naturels peut résulter soit de la mise en œuvre d'une procédure d'expropriation soit d'une cession à l'amiable des parcelles concernées.

Dans la première hypothèse, l'article L. 561-1 du Code de l'environnement prévoit qu'une procédure d'expropriation - qui peut être le cas échéant une procédure accélérée d'extrême urgence - peut être mise en œuvre par l'Etat «lorsqu'un risque prévisible de mouvements de terrain ou d'affaissements de terrain dus à une cavité souterraine ou à une marnière, d'avalanches de crues torrentielles ou à montée rapide ou de submersion marine menace gravement des vies humaines ». En revanche, selon le même article, «ces dispositions ne s'appliquent pas aux cavités souterraines d'origine naturelle ou humaine résultant de l'exploitation passée ou en cours d'une mine ». Par ailleurs, la liste de risques visés par le Code de l'environnement est exhaustive et elle ne concerne pas, notamment, le risque de crues de plaine ${ }^{36}$.

L'article L. 561-1 susvisé conditionne le recours à cette procédure à l'exigence que « les moyens de sauvegarde et de protection des populations s'avèrent plus coûteux que les indemnités d'expropriation ». Il a été jugé, sur ce point, que les autorités compétentes n'ont aucune obligation de chiffrer le coût des mesures de protection, dès lors que le risque naturel en cause rend inenvisageable de telles mesures ${ }^{37}$.

Les parcelles en cause peuvent être des terrains non bâtis ${ }^{38}$, et il est important de relever ici que l'indemnisation qui est assurée par le Fonds de prévention des risques naturels majeurs ne prend pas en compte l'existence du risque naturel, ce qui se justifie par la volonté de ne pas léser les propriétaires concernés et de leur permettre de remplacer les biens expropriés. Ce fonds qui est financé par un prélèvement sur les contrats d'assurance multirisques habitation, finance dans les limites de ses ressources les dépenses liées à la limitation de l'accès et à la démolition éventuelle des biens exposés en vue, selon l'article L. 561-3 du Code d'environnement, «d'en empêcher toute occupation future ».

Le même article prévoit la possibilité de l'acquisition amiable par une commune, un groupement de communes ou l'Etat de biens exposés à un risque prévisible, étant précisé que le fonds de prévention des risques naturels majeurs peut également contribuer au financement de ces mesures. Il en va de même pour les constructions à usage d'habitation ou les bâtiments professionnels des entreprises employant au moins vingt salariés ainsi que leur terrain d'assiette, lorsque ces biens ont été endommagés à plus de la moitié de leur valeur à la suite d'une catastrophe naturelle. Dans ce cas, la participation du fonds est limitée à 60000 euros par unité foncière acquise ${ }^{39}$.

Les terrains acquis devront être déclarés inconstructibles dans un délai de trois ans, à défaut de quoi la collectivité publique autre que l'Etat ayant bénéficié d'un financement sera tenue de rembourser le fonds. 


\section{Les dispositifs spécifiques de prévention de certains risques naturels}

En dehors des dispositions générales que l'on vient d'étudier, le code de l'environnement énumère également des dispositifs spécifiques à certains risques naturels. On verra d'abord la question des risques d'inondation - qui constitue le principal risque naturel en France - avant d'étudier les dispositifs spécifiques visant à la prévention des autres types de risques naturels.

\section{A. La prévention des risques d'inondation}

L'article L. 564-1 du Code de l'environnement précise que "l'organisation de la surveillance, de la prévision et de la transmission de l'information sur les crues est assurée par l'Etat». Les autorités publiques peuvent également mettre en œuvre des mesures spécifiques de prévention des inondations.

\section{$1^{\circ}$. Mesures de prévision des crues}

A la suite d'inondations survenues dans le département du Gard en septembre 2002, le dispositif d'annonce des crues a été entièrement réorganisé et réorienté vers la prévision ce qui a été rendu possible par les progrès techniques importants réalisés ces dernières années en matière de modélisation et de télétransmission en temps réel.

Les services d'annonce des crues ont été remplacés par des services de prévision des crues dont les moyens ont été renforcés. Ces services sont coordonnés et bénéficient de l'appui technique du service central d'hydrométrologie et d'appui à la prévision des inondations (SCHAPI) qui a été créé par un arrêté du 2 juin $2003^{40}$.

L'article L. 564-2 du Code de l'environnement prévoit par ailleurs que le préfet coordonnateur de bassin ${ }^{41}$ arrête un schéma directeur de prévisions des crues. Ce schéma, qui doit être révisé tout les dix ans ${ }^{42}$, doit notamment assurer la cohérence des dispositifs que peuvent mettre en place les collectivités territoriales ou leurs groupements afin de surveiller les crues de certains cours d'eau ou zones estuariennes, avec les dispositifs de l'Etat et de ses établissements publics.

Le schéma directeur de prévision des crues est accompagné d'un règlement relatif à la surveillance et à la prévision des crues et à la transmission de l'information sur les crues $^{43}$. Ce document dresse notamment la liste des communes et des groupements de communes qui bénéficient du dispositif de surveillance et de prévision des crues ainsi que les valeurs des précipitations, des hauteurs des cours d'eau, nappes et estuaires ainsi que des débits des cours d'eau à partir desquelles les autorités de police sont informées du risque d'inondation ${ }^{44}$.

Concrètement, la procédure de vigilance crues - qui s'inspire du dispositif de vigilance météorologique - consiste à identifier les risques susceptibles de survenir et de leur affecter une couleur vert, jaune, orange ou rouge en fonction de son intensité. L'information donne lieu à l'établissement d'une carte de vigilance crue, à l'échelle nationale et sur le territoire de chaque service de prévision des crues. Le SCHAPI et les services de prévision des crues établissent également un bulletin national et des bulletins locaux d'information qui apportent des précisions relatives aux phénomènes 
observés et sur leurs conséquences. Ils définissent également des recommandations à destination des usagers.

\section{$2^{\circ}$. Mesures de prévention des inondations}

La prévention du risque d'inondation passe d'abord par l'obligation d'entretenir les cours d'eaux. Cette obligation repose sur les riverains pour les cours d'eaux non domaniaux et sur la collectivité publique propriétaire pour les cours d'eau qui appartiennent au domaine public fluvial ${ }^{45}$.

Le contenu de cette obligation d'entretien est défini par l'article L. 215-14 du Code de l'environnement : «l'entretien régulier a pour objet de maintenir le cours d'eau dans son profil d'équilibre, de permettre l'écoulement naturel des eaux et de contribuer à son bon état écologique ou, le cas échéant, à son bon potentiel écologique, notamment par enlèvement des embâcles, débris et atterrissements, flottants ou non, par élagage ou recépage de la végétation des rives ».

L'article L. 211-7 du même code prévoit par ailleurs que les collectivités publiques peuvent entreprendre l'étude, l'exécution et l'exploitation de tous travaux, actions, ouvrages ou installations présentant un caractère d'intérêt général ou d'urgence notamment pour l'entretien et l'aménagement des cours d'eau, la défense contre les inondations et la mise en place et l'exploitation de dispositifs de surveillance des cours d'eau. Ces dispositions renvoient aux articles L. 151-36 et suivants du Code rural qui exigent que ces opérations soient déclarées d'intérêt général ou d'urgence par arrêté ministériel ou préfectoral après enquête publique. Toutefois, cette enquête n'est pas obligatoire dans certains cas, en particulier en cas de situation de péril imminent ou après une inondation déclarée catastrophe naturelle.

Selon l'article L. 215-15 du Code de l'environnement ces opérations peuvent être "menées dans le cadre d'un plan de gestion établi à l'échelle d'une unité hydrographique cohérente et compatible avec les objectifs du schéma d'aménagement et de gestion des eaux lorsqu'il existe».

Les dépenses exposées par les collectivités publiques peuvent être partiellement mises à la charge des personnes qui ont rendu les travaux nécessaires ou qui y trouvent intérêt ${ }^{46}$. Toutefois les propriétaires concernés disposent d'un droit de délaissement, qui va contraindre la personne publique concernée à acquérir leur bien dans un délai de deux ans, lorsque le montant de la participation aux travaux est supérieur au tiers de la valeur avant travaux du bien immobilier qui en bénéficie ${ }^{47}$. Le fonds de prévention des risques naturels majeurs peut également contribuer au financement de ces travaux lorsque ce sont des collectivités territoriales ou leurs groupements qui en assurent la maîtrise d'ouvrage, pour les communes couvertes par un PPRNP.

En dehors des mesures d'entretien des cours d'eaux, l'article. L. 211-2 du Code de l'environnement précise que «des servitudes d'utilité publique peuvent être instituées à la demande de l'Etat, des collectivités territoriales ou de leurs groupements sur des terrains riverains d'un cours d'eau ou de la dérivation d'un cours d'eau, ou situés dans leur bassin versant, ou dans une zone estuarienne ». Ces servitudes vont par exemple permettre de créer des zones de rétention temporaire des eaux de crues ou de ruissellement par des aménagements permettant d'accroître artificiellement leur capacité de stockage de ces eaux, afin de réduire les crues ou les ruissellements dans des secteurs situés en aval. L'instauration de ces servitudes donne droit à 
indemnisation pour les propriétaires lorsqu'elles créent un préjudice matériel, direct et certain. Ces indemnités sont à la charge de la collectivité qui a demandé l'institution de la servitude et elles sont fixées, à défaut d'accord amiable par le juge de l'expropriation. Le propriétaire de la parcelle grevée d'une servitude peut demander à la collectivité qui a sollicité son institution d'en faire l'acquisition. Ce droit de délaissement se fait conformément aux dispositions des articles L. 230-1 et suivants du Code de l'urbanisme. Enfin, conformément à l'article L. 221-12 du Code de l'environnement, les communes et les établissements publics de coopération intercommunale peuvent instituer le droit de préemption urbain de l'article L. 211-1 du Code de l'urbanisme dans les zones soumises à ces servitudes ou déléguer ce droit à la collectivité qui a demandé l'institution de la servitude. Cette procédure va permette à la personne publique concernée d'acquérir en priorité les biens immobiliers mis en vente par une personne privée.

\section{B. Mesures spécifiques à la prévention des autres risques naturels}

Ces mesures spécifiques concernent les risques de mouvements de terrains, les risques sismiques et les risques d'avalanche.

\section{$1^{\circ}$. Mesures de prévention des risques de mouvements de terrain}

Selon la définition que l'on peut trouver sur le site internet du ministère de l'écologie, du développement durable, des transports et du logement « les mouvements de terrain regroupent un ensemble de déplacements, plus ou moins brutaux, du sol ou du soussol, d'origine naturelle ou anthropique... Les déplacements peuvent être lents (quelques millimètres par an) ou très rapides (quelques centaines de mètres par jour) ${ }^{48}$.

Environ 7000 communes françaises sont concernées par un risque de mouvements de terrain dont un tiers avec un niveau de gravité fort vis-à-vis des populations ${ }^{49}$.

L'article L. 536-6 II du Code de l'environnement prévoit d'abord que toute personne qui a connaissance de l'existence d'une cavité souterraine ou d'une marnière dont l'effondrement est susceptible de porter atteinte aux personnes ou aux biens, ou d'un indice susceptible de révéler cette existence, en informe le maire. Le maire doit ensuite communiquer, sans délai, au préfet et au président du conseil général, les éléments dont il dispose à ce sujet. Sur la base de ces éléments, le préfet tient à jour une liste des communes pour lesquelles il existe une cavité souterraine ou une marnière et de celles où il existe une présomption réelle et sérieuse de l'existence d'une telle cavité. L'article L. 563-6 du Code de l'urbanisme précise quant à lui que les communes et leurs groupements peuvent élaborer des cartes délimitant les sites où sont situées des cavités souterraines et des manières susceptibles de provoquer l'effondrement du sol.

Dans le cadre de ses pouvoirs de police, le maire peut également prescrire « de clore ou de combler les carrières, argilières, sablonnières, ballastières, marnières, fosses à chaux, glaisières, puits de mine, trous de fouille ou trous provenant du déracinement des souches $\|^{50}$. Il peut également « marquer par des signes visibles, pour en empêcher l'approche, les trous faits dans la glace aux endroits indiqués par l'autorité ${ }^{51}$.

Enfin, l'article L. 561-3 du Code de l'environnement prévoit que le fonds de prévention des risques naturels majeurs finance à hauteur de $30 \%$ les opérations de reconnaissance des cavités souterraines et des marnières, dont les dangers pour les constructions ou les vies humaines sont avérés, ainsi que le traitement ou le comblement des cavités 
souterraines et des marnières qui occasionnent des risques d'effondrement du sol menaçant gravement des vies humaines, dès lors que ce traitement est moins coûteux que l'expropriation.

\section{$2^{\circ}$. Mesures de prévention des risques sismiques}

Des avancées scientifiques et l'adoption d'un nouveau code européen de construction parasismique - l'Eurocode 8 (ou EC8) - ont conduit à la révision du zonage sismique qui existait depuis 1991 : du fait de cette évolution le nombre de communes concernées par les risques sismiques est passé de 5000 à 12000 . Ce nouveau zonage sismique est en vigueur depuis le 1er mai $2011^{52}$ et il distingue cinq zones de sismicité numérotées de 1 à 5 : sismicité très faible, faible, modérée, moyenne et sismicité forte ${ }^{53}$.

Ce nouveau zonage a été accompagné de nouveaux textes réglementaires fixant les règles de construction parasismiques, précisant l'article R. 563-5 du Code de l'environnement. Un arrêté du 22 octobre 2010 définit les règles applicables aux bâtiments de la classe dite « à risque normal $»^{54}$, ce qui vise selon l'article R. 563-3 du Code de l'environnement « les bâtiments, équipements et installations pour lesquels les conséquences d'un séisme demeurent circonscrites à leurs occupants et à leur voisinage immédiat ». Un arrêté du 24 janvier 2011, qui n'entrera en vigueur que le 1er janvier 2013, s'applique aux installations présentant un "risque spécial», ce qui concerne en application de l'article R. 563-6 du même code «les bâtiments, les équipements et les installations pour lesquels les effets sur les personnes, les biens et l'environnement de dommages même mineurs résultant d'un séisme peuvent ne pas être circonscrits au voisinage immédiat des dits bâtiments, équipements et installations $»{ }^{55}$. Cette seconde catégorie concerne plus spécialement les installations classées dites « Seveso ».

Par ailleurs, l'article L. 563-1 du Code de l'environnement prévoit que si un plan de prévention des risques naturels prévisibles est approuvé dans une zone de sismicité, il peut éventuellement fixer des règles plus adaptées à la nature des risques encourus que celles résultant de la réglementation susvisée. Mais dans ce cas il est nécessaire, comme le précise l'article R. 563-8 du même code "qu'elles garantissent une protection au moins égale à celle qui résulterait de l'application de ces dernières règles ${ }^{56}$.

Enfin, il faut relever l'existence d'un plan national de prévention du risque sismique dit "plan séisme ", mis en œuvre entre 2005 et 2011, dont l'objectif principal était de réduire la vulnérabilité de la France face au risque sismique. Ce plan comportait quatre axes : formation, information et connaissance du risque; amélioration de la prise en compte du risque sismique dans les constructions; concertation, coopération et communication; prévention du risque de tsunami. Ces thématiques ont donné lieu à la mise en place de 80 actions opérationnelles qui ont été pilotées par les directions régionales de l'environnement. Le plan séisme est complété, pour des zones représentant les risques plus importants que sur territoire métropolitain, par un plan séisme Antilles dont l'objectif principal est la réduction de la vulnérabilité sismique du bâti existant, soit par confortement parasismique soit par démolition et reconstruction.

\section{$3^{\circ}$. Mesures de prévention du risque d'avalanches}

Dans ce domaine également, il a fallu attendre une catastrophe majeure, celle de l'avalanche qui emporta le 10 février 1970 le chalet de l'UCPA à Val-d'Isère pour que le 
risque d'avalanches fasse l'objet de mesures de prévention. Cette prise de conscience s'est traduite par l'élaboration de plans des zones exposées aux avalanches (PZEA) ${ }^{57}$, auxquels se sont substitués les documents plus généraux que constituent les plans d'exposition aux risques et les plans de prévention des risques naturels prévisibles.

L'article L. 563-2 du Code de l'environnement prévoit qu'en l'absence de plan de prévention des risques naturels prévisibles, les documents d'urbanisme ainsi que les décisions d'urbanisme "tiennent compte des risques naturels spécifiques à ces zones, qu'il s'agisse de risques préexistants connus ou de ceux qui pourraient résulter des modifications de milieu envisagées ». Il doit également être tenu compte de ces risques pour la délivrance des autorisations relatives aux remontées mécaniques

D'autres mesures à caractère préventif existent également. D'une part un bulletin d'estimation du risque d'avalanche est régulièrement édité par Météo-France. D'autre part, le maire peut établir un plan d'intervention et de déclenchement des avalanches qui permet de déclencher artificiellement des avalanches en ayant recours aux explosifs.

\section{CONCLUSION}

En guise de conclusion, on peut observer que l'architecture des dispositifs de prévention des risques naturels est extrêmement complexe, ce qui s'explique principalement par la variété des de ces risques qui induit la diversité des réglementations spécifiques, leur extrême technicité qui est parfois difficile à comprendre pour un juriste et la difficulté corrélative de mettre en œuvre une réglementation globale. Quoi de commun en effet entre une commune des Hautes-Alpes soumise à un risque d'avalanche, une commune de Guadeloupe située en zone de force sismicité ou encore une commune soumise à un risque d'inondation? Un autre facteur important de complexité résulte du caractère évolutif de la réglementation, qui est elle même liée à deux éléments : l'amélioration constante des techniques qui permettent de mieux appréhender les risques et de limiter les conséquences de leur survenance mais également l'effet sur l'opinion des catastrophes naturelles et leurs conséquences sur la réglementation.

Comme on l'a vu, et ce n'est qu'un exemple, ce sont des inondations survenues dans le Gard en 2002 qui ont conduit à repenser les règles de prévention contre les crues. Plus récemment, la tempête Xynthia survenue en 2010 à permis de prendre conscience de l'insuffisance des dispositifs de prévention contre les risques de submersion marine. Elle a aboutit à une remise en cause de l'urbanisation du littoral, à repenser les systèmes d'alerte et à lancer un «plan digue » qui devrait aboutir à la reconstruction ou au renforcement de 1200 kilomètres de digues, mais également à la suppression de celles qui n'apparaissent pas comme essentielles ${ }^{58}$. Le constat d'une couverture insuffisante des territoires par des plans de prévention des risques naturels littoraux a également conduit à accélérer leur déploiement, l'ensemble des communes concernées devant être couvertes d'ici $2014^{59}$.

Cet exemple récent démontre que la réglementation actuelle est loin d'être achevée : l'évolution des techniques, la survenance inévitable de nouvelles catastrophes naturelles qui tendront à démontrer son inadaptation aux réalités de terrain conduiront nécessairement à de nouvelles évolutions. 


\section{NOTES}

1. http://www.senat.fr/rap/l02-154/I02-1547.html\#toc63

2. V. en particulier le décret impérial du 15 octobre 1810 relatif aux manufactures et ateliers qui répandent une odeur insalubre ou incommode.

3. V. C. urb. , art. L. 121-1 s. et R. 121-1 s. et plus spécifiquement Art. L. 121-1-1 s. et R. 122-1 s.

4. V. C. urb., art. L. 121-1 s. et R. 121-1 s. et plus spécifiquement Art. L. 123-1 s. et R. 123-1 s.

5. Loi $n^{\circ}$ 95-101, relative au renforcement de la protection de l'environnement.

6. Ordonnance $n^{\circ} 2000-914$ du 18 septembre 2000 relative à la partie législative du Code de l'environnement.

7. Ces plans avaient été créés par la loi n82-600 du 13 juillet 1982

8. Loi $n^{\circ} 2003-699$

9. L'eau et son droit : EDCE, 2010, V. p. 183.

10. C. env., art. L 562-3. V. par ailleurs sur les modalités formelles de présentation des registres d'enquête C. env. art. R. 562-8.

11. C. env., art. R. 562-1.

12. C. urb., art. L. 126-1.

13. CAA Bordeaux, 28 mai 2003, SCl Fier Les Portes la Grande Jetée : Rev. jur. env. 2005, p. 243, note Monédiaire.

14. Circ., 20 juillet 2006 du Ministre des transports, de l'équipement, du tourisme et de la mer et de la ministre de l'écologie et du développement durable, relative à la protection de l'environnement littoral : BOMEDD, n 14, 2006.

15. TA Nice, 18 mars 2003, de Panisse-Passis et SCl I'Aspre Redon c/ préfet AlpesMaritimes : Environnement 2003, 71, note Trouilly.

16. CE, 14 février 2007, Communauté de communes de Blangy-Pont-l'Evêque : JCP A 2007, 2170, note Billet ; Environnement 2007, 88, note Trouilly.

17. C. env., art. L.562-1 II, $1^{\circ}$ et $2^{\circ}$.

18. C. env., art. L. 562-1 II, $3^{\circ}$.

19. C. env. ,art. L. 562-1 III et R. 562-3 $3^{\circ}$ b.

20. C. env. ,art. R. 562-5.

21. C. env. ,art. R. 561-8 $8^{\circ}$. V. également Circulaire, 23 février 2005 : BOMEDD, $\mathrm{n}^{\circ} 71$, 2005.

22. CAA Nancy, 10 avril 2003, Société Le Nid : Environnement 2003, 70, note Trouilly.

23. CE, 3 juillet 1998 : Rec. p. 288, concl. Abraham.

24. CE, 29 décembre 2004, Société d'aménagement des coteaux Saint-Blaine: Rec. p. 478 ; Dr. Adm., 45, note Mahinga; AJDA 2005, p. 423, note Landais et Lenica; Environnement 2005, 18, note Trouilly.

25. JO 31 juillet 2003.

26. C. env., art. R. 565-2.

27. C. env., art. R. 565-4.

28. C. env., art. R. 565-2.

29. C. env., art. R. 565-3.

30. CE, 25 juillet 2007, Leberger et Cortie : Dr. Adm. 2007, 159, note Melleray ; JCP A 2007, 2319, note Ngampio-Obélé-Bélé ; RJEP décembre 2007, 19, concl. Chauvaux.

31. CE, 6 avril 1998, SARL Anciens établissements Ousteau et Cie : Rec. tables, p. 1062. 
32. Cass. $1^{\text {ère }}$ civ., 28 novembre 2007, n06-19405 : Bull. civ. I, nº 372.

33. CE, 27 juin 2005, Ville Orléans : Rec. tables, p. 757 et 995.

34. CE, 7 février 2003, Commune de Beugnatre : Rec. tables, p. 678.

35. CE, 10 octobre 2005, Commune de Badinières : Rec. p. 426.

36. Circulaire $n^{\circ} 96-53,10$ juillet 1996, relative à l'expropriation des biens exposés à certains risques naturels majeurs menaçant gravement des vies humaines.

37. CE, 7 avril 1999, Association "Vivre et rester au pays" : AJPI 1999, p. 766, note Hostiou.

38. CE, 16 février 2004, Ministre de l'économie c/ SARL Le Panoramic et SCI les Rameirols : Environnement 2004, 69, note Benoît

39. A., 12 janv. 2005, relatif aux subventions accordées au titre du financement par le fonds de prévention des risques naturels majeurs de mesures de prévention des risques naturels majeurs : J0, 15 Janvier 2005.

40. JO, 5 juin 2003.

41. V. Décret $n^{\circ} 2005-636$ du 30 mai 2005 relatif à l'organisation de l'administration dans le domaine de l'eau et aux missions du préfet coordonnateur de bassin.

42. C. env. ,art. R. 564-5.

43. C. env., art. 564-7.

44. C. env., art. R. 564-8.

45. CGPPP, art. L. 2124-11.

46. C. rur., art. L. 151-36.

47. Ibid.

48. http://www.developpement-durable.gouv.fr/Le-risque-mouvement-de-terrain.html

49. http://www.driea.ile-de-france.developpement-durable.gouv.fr/le-risque-mouvements-deterrain-r1642.html

50. CGCT, art. L. 2542-7 $1^{\circ}$.

51. CGCT, art. L. 2542-7 $2^{\circ}$.

52. C. env., art R. 563-1 et D. 563-8-1.

53. C. env., art. R. 563-4.

54. JO, 24 octobre 2010.

55. JO, 31 mars 2011.

56. V. par exemple CAA Marseille, 16 mai 2007, Commune de Meyrargue, req. n04MA00587.

57. Circulaire $n^{\circ}$ 74-201, 5 décembre 1974.

58. http://www.developpement-durable.gouv.fr/Presentation-du-plan-de.html

59. Circulaire du Ministre de l'écologie, du développement durable, des transports et du logement, 2 août 2011.

\section{RÉSUMÉS}

La prévention des risques naturels en France fait l'objet d'une réglementation général et de réglementations spécifiques adaptées à certains types de risques. Les règles en vigueur, qui sont caractérisées par leur complexité et leur grande technicité sont loin d'être achevées : l'évolution des techniques, l'apparition inévitable de nouvelles catastrophes naturelles mettront en lumières des lacunes qui conduiront inévitablement à de nouvelles règles.

Prevention of natural hazards in France is the object of devices that consist of a general regulation for all risks and specific regulations to certain risks in particular. Current regulations, 
which is characterized by both its complexity and a large technicality is far from over: the evolution of techniques, the inevitable emergence of new natural disasters tend to demonstrate its failure to adapt to realities on the ground and will inevitably lead to new developments.

\section{AUTEUR}

PIERRE TIFINE

Professeur de droit public à l'université de LorraineDirecteur adjoint de l'IRENEE 